predominantly heterosexual. The aim of this study conducted in Cotonou, Benin, was to determine factors associated with unprotected sex among female sex workers (FSW), an important core group for the transmission of HIV and sexually transmitted infections (STI).

Methods We have recruited and followed 396 FSW in a reference center for STI treatment. Condom use was assessed at enrolment and 6 months thereafter. FSW were asked how often they have used condoms with their clients during the last 7 days of work. During the study, FSW were encouraged to always use condoms with their clients. A generalised estimating equation model for binomial distribution with repeated measures was used to isolate factors independently associated with failure to use condoms during any sexual act.

Results The cohort comprised 1) 149 HIV-positive FSW, of which 52 were treated with antiretroviral therapy (ART) and 97 were not yet eligible for ART and 2) 247 HIV-negative subjects. Mean age (standard deviation; sd) and mean number of clients (sd) during the last 7 days of work were, respectively, 33.8 (9.3) years and 28.4 (26.1) clients. The majority of the FSW (55.3\%) did not practice in brothels or equivalent set ups. At enrolment, 5.6\% of the FSW reported that they have never used condoms during the last week of work. The proportion of FSW with full use of condoms during the last 7 days of work increased from $77.8 \%$ at enrolment to $90.6 \%$ 6 months after. First assessment of condom use (enrolment into the study) [RR 2.17; 95\% CI 1.44\% to $3.26 \%$ ], not working in a brothel (RR 2.22; $95 \%$ CI $1.39 \%$ to $3.54 \%$ ), having a relatively low monthly income (RR $1.63 ; 95 \%$ CI $1.04 \%$ to $2.57 \%$ ) and older age (RR 1.50; $95 \%$ CI 1.03 to $2.21 \%$ ) were independently associated with failure to always use condoms with the clients. Being HIV-infected or being treated with ART was not associated with the issue of interest. The reasons reported by the FSW for not using condoms during the last sexual act were dominated by the refusal of the client (46.0\%).

Conclusion As suggested by our results, interventions promoting condom use during any commercial sexual act are fruitful and need to target women outside brothels, economically more vulnerable and older FSW as well as their clients.

\section{1-S08.04 CENTRAL AMERICAN SURVEILLANCE SURVEY OF SEXUAL BEHAVIOUR AND PREVALENCE OF HIV/STIS IN VULNERABLE POPULATIONS: FEMALE SEX WORKERS, NICARAGUA, 2009}

doi:10.1136/sextrans-2011-050109.46

\author{
${ }^{1} \mathrm{~S}$ Delgado, ${ }^{1} \mathrm{~B}$ Alvarez, ${ }^{1} \mathrm{~J}$ Goins, ${ }^{1} \mathrm{~L} \mathrm{M}$ Romero, ${ }^{2} \mathrm{E} J \mathrm{~B}$ Acevedo, ${ }^{3} \mathrm{G}$ Paz-Bailey, \\ ${ }^{1} S$ Morales. 'Universidad del Valle de Guatemala, Guatemala City, Guatemala; \\ ${ }^{2}$ Ministerio de Salud de Nicaragua, Nicaragua; ${ }^{3}$ Tephinet, USA
}

Background From 2001 to 2009 the estimated number of adults and children living with HIV in Nicaragua nearly doubled from 3700 to 6900. In Central America, the HIV epidemic is primarily concentrated in a few populations, including female sex workers (FSWs), who also bear a substantial burden of other sexually transmitted infections (STIs). This study reports results from the Central American Surveillance Survey of Sexual Behaviour and Prevalence of HIV/STIs in Vulnerable Populations, which was conducted among FSWs in Nicaragua in 2009.

Methods We conducted a comprehensive sampling of FSWs in the Nicaraguan cities of Managua $(N=618)$ and Chinandega $(N=214)$ in 2009. Utilising a behavioural surveillance survey (BSS) approach within the framework of second generation HIV surveillance, we conducted a cross-sectional evaluation of HIV prevalence and incidence, prevalence of other STIs, and relevant behavioural and contextual factors.

Results Estimated HIV prevalence among FSWs was 1.8\% (95\% CI $0.9 \%$ to $3.2 \%$ ) in Managua and $2.4 \%$ (95\% CI: $0.8 \%$ to $5.4 \%$ ) in
Chinandega. Estimated annual HIV incidence among all participating FSWs was $0.8 \%$ (95\% CI: $0.0 \%$ to $2.0 \%$ ). In Managua $36.5 \%$ of FSWs (95\% CI: $32.7 \%$ to $40.5 \%$ ) and in Chinandega $51.4 \%$ of FSWs (95\% CI: $44.5 \%$ to $58.3 \%$ ) had received an HIV test in the 12 months prior to the study. Substantial prevalence of infection with herpes simplex virus type 2 (HSV-2) was found among FSWs in both Managua (75.7\%; $95 \%$ CI: $72.1 \%$ to $79.0 \%$ ) and Chinandega (83.5\%; 95\% CI: $77.8 \%$ to $88.2 \%$ ). All FSWs with HIV infection also had HSV-2 infection. Considerable but variable prevalence of other STIs was also present among FSWs participating in the study (Abstract O1-S08.04 table 1). Consistent condom use during the previous 30 days among FSWs varied markedly according to type of sexual partner, and was highest with clients $(89.9 \%$; $95 \%$ CI: $87.5 \%$ to $91.7 \%$ ), lower with occasional partners (61.0\%; $95 \%$ CI: $50.9 \%$ to $70.3 \%)$, and lowest with stable partners $(12.7 \%$; $95 \% \mathrm{CI} ; 9.7 \%$ to $16.3 \%)$.

Abstract 01-S08.04 Table 1 Prevalence of HIV, HSV-2, and other STIs among FSWs in Managua and Chinadega, Nicaragua, 2009

\begin{tabular}{|c|c|c|c|c|c|c|}
\hline & \multicolumn{3}{|c|}{ Managua } & \multicolumn{3}{|c|}{ Chinandega } \\
\hline & $\mathbf{N}$ & $\mathbf{n}$ & $\%(95 \%$ CI) & $\mathbf{N}$ & $\mathbf{n}$ & $\%(95 \% \mathrm{Cl})$ \\
\hline HIV & 613 & 11 & $1.8(0.9$ to 3.2$)$ & 211 & 5 & $2.4(0.8$ to 5.4$)$ \\
\hline HSV-2 & 613 & 464 & 75.7 (72.1 to 79.0$)$ & 212 & 177 & 83.5 (77.8 to 88.2 ) \\
\hline Syphilis & 618 & 43 & 7.0 (5.1 to 9.3$)$ & 213 & 1 & $0.5(0.0$ to 2.6$)$ \\
\hline Active syphilis & 618 & 23 & $3.7(2.4$ to 5.5$)$ & 213 & 0 & $0.0(0.0$ to 1.7$)$ \\
\hline Bacterial vaginosis & 570 & 209 & 36.7 (32.7 to 40.8 ) & 182 & 74 & 40.7 (33.5 to 48.2 ) \\
\hline Chlamydia trachomatis & 605 & 125 & 20.7 (17.5 to 24.1$)$ & 188 & 19 & $10.1(6.2$ to 15.3$)$ \\
\hline Mycoplasma genitalium & 605 & 102 & $16.9(14.0$ to 20.1$)$ & 188 & 28 & 14.9 (10.1 to 20.8$)$ \\
\hline Neisseria gonorrhoeae & 605 & 27 & $4.5(3.0$ to 6.4$)$ & 188 & 7 & 3.7 (1.5 to 7.5$)$ \\
\hline Trichomonas vaginalis & 605 & 182 & 30.1 (26.5 to 33.9$)$ & 188 & 91 & 48.4 (41.1 to 55.8 ) \\
\hline
\end{tabular}

Conclusions Our estimate of HIV prevalence among FSWs in Managua is higher than the $0 \%(97.5 \% \mathrm{CI}: 0.0 \%$ to $1.1 \%) \mathrm{HIV}$ prevalence reported for this population in the 2001-2002 Estudio Multicéntrico. Similarly, we report an annual HIV incidence estimate that is higher than the $0 \%$ annual HIV incidence reported in the 2001-2002 study. Compared to the Estudio Multicéntrico, we found slightly higher rates of consistent condom use with stable partners, and substantially higher rates of consistent condom use with clients.

\section{1-S08.05 RECENT TRENDS IN STIS AND HIV AMONG FEMALE SEX WORKERS AND THEIR CLIENTS IN INDIA: RESULTS FROM REPEATED CROSS-SECTIONAL SURVEYS}

doi:10.1136/sextrans-2011-050109.47

${ }^{1} \mathrm{R}$ Adhikary, 'S Ramanathan, ${ }^{1} \mathrm{~A}$ Gautam, ${ }^{1} \mathrm{P}$ Goswami, ${ }^{1} \mathrm{~L}$ Ramakrishnan, ${ }^{1} \mathrm{~S}$ Kallam ${ }^{2} \mathrm{M}$ M Mainkar, ${ }^{3} \mathrm{G}$ N V Brahmam, ${ }^{4} \mathrm{~T}$ Subramanaian, ${ }^{2} \mathrm{R}$ S Paranjape. ${ }^{1} \mathrm{FHI}$, New Delhi, India; ${ }^{2}$ National AIDS Research Institute NARI, India; ${ }^{3}$ National Institute of Nutrition NIN, India; ${ }^{4}$ National Institute of Epidemiology NIE, India

Background HIV transmission in India is concentrated among highrisk groups. Female sex workers (FSWs) and Clients of FSWs are the most vulnerable groups at risk for HIV. This paper presents the prevalence of sexually transmitted infections (STIs) and HIV among FSWs and their clients in the states of Andhra Pradesh (AP), Tamil Nadu (TN) and Maharashtra (MH) from repeated, cross-sectional, bio-behavioural surveys.

Methods Data from two rounds of Integrated Behavioural and Biological Assessments conducted in 2005 (R1) and 2009 (R2) among 15632 FSWs (R1-7828, R2-7804) and 9624 clients of FSWs (R1-4821, R2-4803) were analysed to observe the changes in STI and HIV prevalence among each group. Respondents' behaviour was 
assessed and blood and urine samples were collected to test for STIs [Chlamydia trachomatis (CT), Neisseria gonorrhoeae (NG), syphilis and HIV]. Analysis at both the aggregate and state level was done. Adjusted ORs were obtained by including all socio-demographic variables associated with rounds of survey in multivariate models. Results At the aggregate level, among FSWs, prevalence of syphilis declined from $10.8 \%$ in $\mathrm{R} 1$ to $5.0 \%$ in $\mathrm{R} 2$ (AOR 0.39, $\mathrm{p}=0.001$ ) and no significant change was observed in prevalence of HIV, NG or CT. Among FSW clients, STI prevalence was low in R1 (syphilis $4.7 \%$, NG $0.4 \%$ and CT $2.4 \%$ ) and no significant change was observed in R2. The state-wide trend shows that for FSWs in AP, a significant decline occurred in prevalence of both HIV (AOR 0.68, p<0.01) and syphilis (AOR $0.39, p<0.001$ ) whereas in $\mathrm{TN}$, only syphilis prevalence declined (AOR 0.20, $\mathrm{p}<0.001$ ). The trend in $\mathrm{MH}$ differs from the other two states. In $\mathrm{MH}$, prevalence of bacterial STIs (syphilis, NG and CT) declined significantly whereas HIV prevalence increased significantly (AOR 1.29, $\mathrm{p}=0.04$ ). Among FSW clients, STI prevalence was low in $\mathrm{MH}$ (syphilis $4.3 \%$, NG $0.7 \%$, and CT $4.2 \%$ ) and TN (syphilis $4.1 \%$, NG $0.0 \%$, and CT $0.8 \%$ ) in R1 and remained unchanged in R2. However, a significant decline occurred in HIV prevalence in both states [MH: AOR-0.25, $p=0.002$; TN: AOR-2.3, $\mathrm{p}<0.05]$. In $A P$, only prevalence of syphilis (AOR $0.18, p<0.001$ ) declined and no change was observed in other STIs.
Conclusion The prevalence of syphilis declined among both FSWs and their clients in all three states whereas no change was seen in other bacterial STI prevalence (NG or CT). Among FSW clients in $\mathrm{MH}$ and TN, STI prevalence remained unaltered. Tailored and sustained programmatic efforts are needed to address the disparity in prevalence of STIs and HIV in the three states.

\section{1-S08.06 VARIABILITY IN THE NUMBERS AND TYPES OF SEX PARTNERS OF FEMALE SEX WORKERS AND ITS IMPACT ON HIV PREVALENCE IN SOUTHERN INDIA}

doi:10.1136/sextrans-2011-050109.48

${ }^{1} \mathrm{~K}$ Deering, ${ }^{1} \mathrm{~J}$ Shoveller, ${ }^{2} \mathrm{M}$ Pickles, ${ }^{3} \mathrm{~S}$ Shaw, ${ }^{3} \mathrm{~S}$ Moses, ${ }^{3} \mathrm{~J}$ Blanchard, ${ }^{4} \mathrm{R}$ Washington, ${ }^{4} \mathrm{~B} \mathrm{M}$ Ramesh, ${ }^{4} \mathrm{~S}$ Isac, ${ }^{2} \mathrm{M} \mathrm{C}$ Boily. ${ }^{1}$ University of British Columbia, Vancouver, Canada; ${ }^{2}$ Imperial College, UK; ${ }^{3}$ University of Manitoba, Winnipeg, Canada; ${ }^{4}$ Karnataka Health Promotion Trust, Bangalore, India

Background Heterogeneity in the structure of sex work within and across female sex worker (FSW) populations may be an important determinant of the spread of HIV. We characterised heterogeneity in sexual structure and assessed how it influenced HIV prevalence on a population level in southern India.

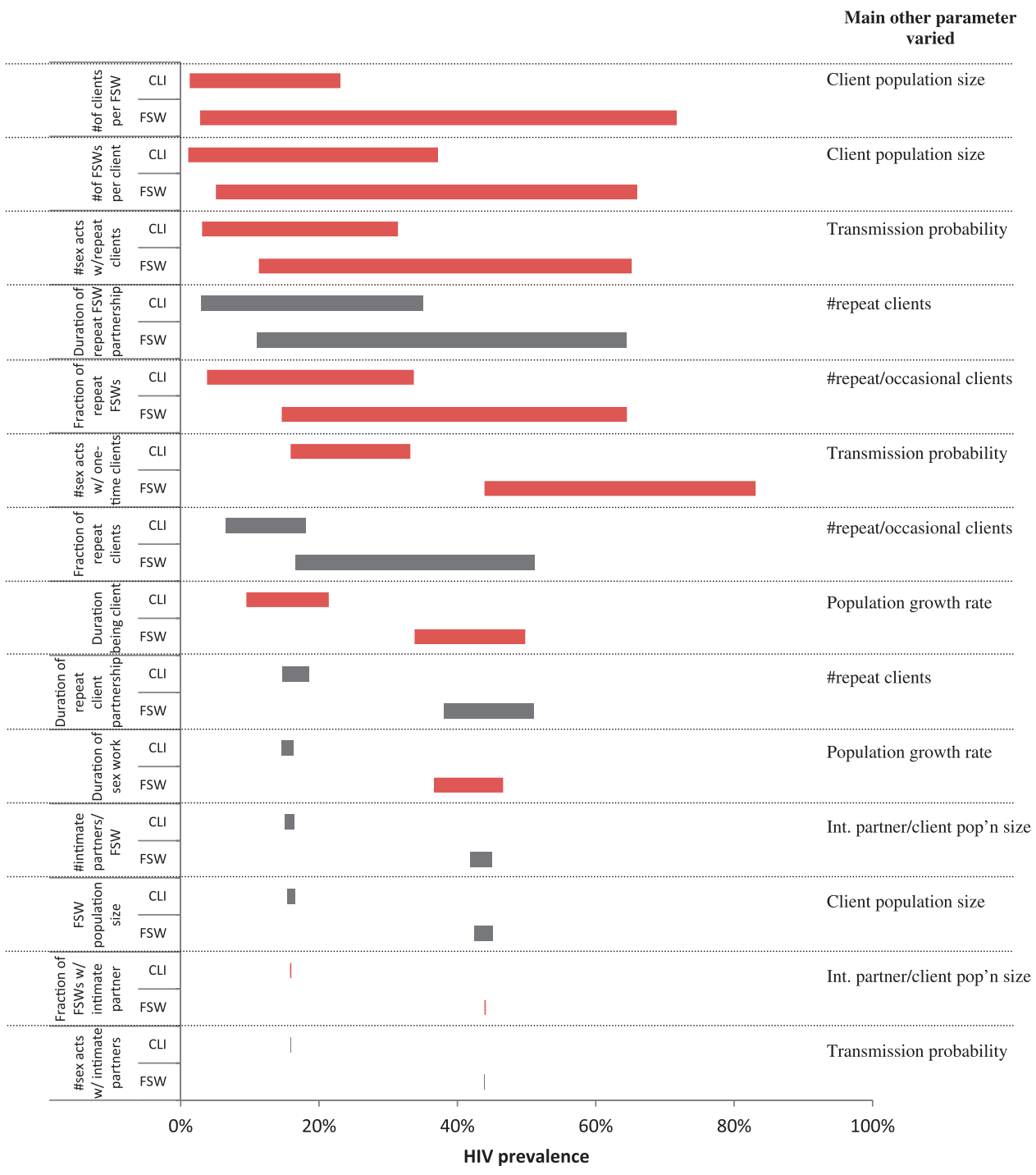

Abstract 01-S08.06 Figure 1 Impact of varying each sexual structure parameter through a wide range (half to double the baseline value) on peak HIV prevalence in district A. . ${ }^{1}$ Red bars=a positive correlation and grey bars=a negative correlation. Bars are arranged in the order of the absolute impact of each parameter on peak HIV prevalence. 